\title{
A rare complication of video-assisted thoracoscopic surgery: lung herniation retrospective case series of three patients and review of the literature
}

\author{
Güntuğ Batıhan ${ }^{1}$, Demet Yaldız², Kenan Can Ceylan ${ }^{1}$ \\ ${ }^{1}$ Department of Thoracic Surgery, Dr Suat Seren Chest Diseases and Surgery Medical Practice and Research Center, \\ University of Health Sciences, Izmir, Turkey \\ 2Department of Thoracic Surgery, Faculty of Medicine, Manisa Celal Bayar University, Manisa, Turkey
}

Videosurgery Miniinv 2020; 15 (1): 215-219

DOI: https://doi.org/10.5114/wiitm.2019.87937

\begin{abstract}
Introduction: Lung herniation is defined as a protrusion of the lung parenchyma with its pleura through the intercostal space. It is a rare condition and usually occurs after thoracic trauma and surgical interventions. A few cases of lung herniations have been reported after video-assisted thoracoscopic surgery (VATS) but only two cases have been reported after VATS lobectomy.

Aim: The VATS procedure has become the dominant method of lung cancer surgery, but there is no case series about the complications of lung herniation in the literature. We aim to define some risk factors and possible ways of prevention of lung herniation after VATS resection.

Material and methods: This study retrospectively analyses 650 (550 anatomic, 100 non-anatomic sublobar resections) patients who underwent lung resections for lung cancer in our department between 2012 and 2018. We detected lung herniation in 3 patients after VATS resection.

Results: Asymptomatic lung hernias may be managed by close observation but because of the risk of incarceration of the pulmonary parenchyma, surgery is often necessary. The main steps of treatment involve: identification of the hernia, freeing of all adhesions, reduction of the lung tissue back into the thoracic cavity and repairing the defect of the chest wall.

Conclusions: By this retrospective case series, we defined some patient-related and surgeon-related risk factors and some basic recommendations for prevention of this complication.
\end{abstract}

Key words: complication, lung herniation, video-assisted thoracoscopic surgery.

\section{Introduction}

Lung herniation is a rare condition involving protrusion of the lung parenchyma with its pleura caused by a defect of the thoracic wall. Herniation usually occurs after thoracic trauma and surgery, but spontaneous pulmonary herniation has been reported previously [1]. As video-thoracoscopic surgery proce- dures evolve, different and rare complications such as lung herniation have begun to occur. It was reported as a complication of anterior thoracotomy before but there are only a few reported cases of lung herniation as a complication of video-thoracoscopic surgery in the literature. The first case was reported as a complication of video-assisted thoracoscopic surgery (VATS) by van den Brink et al. in 1995 and to date, only 8 cas-

\section{Address for correspondence}

Güntuğ Batıhan MD, Department of Thoracic Surgery, Dr Suat Seren Chest Diseases and Surgery Medical Practice and Research Center, University of Health Sciences, Izmir, Turkey, e-mail: gbatihan@hotmail.com 
es of lung herniation as a complication of VATS have been described in the literature [2-8].

We present three cases of lung herniation as a complication of video-thoracoscopic surgery. It is the first case series in the literature according to our knowledge.

\section{Aim}

The aim of this study is to evaluate risk factors of lung herniation after VATS resection and to define some recommendations for preventing this complication.

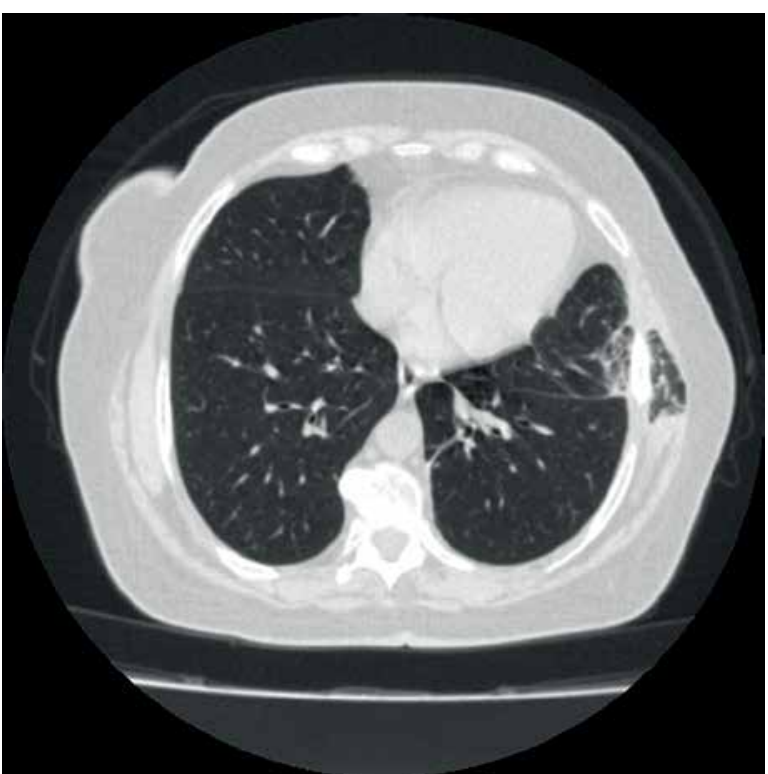

Photo 1. Chest CT revealed protrusion of a small part of left lower lobe through the $7^{\text {th }}$ intercostal space

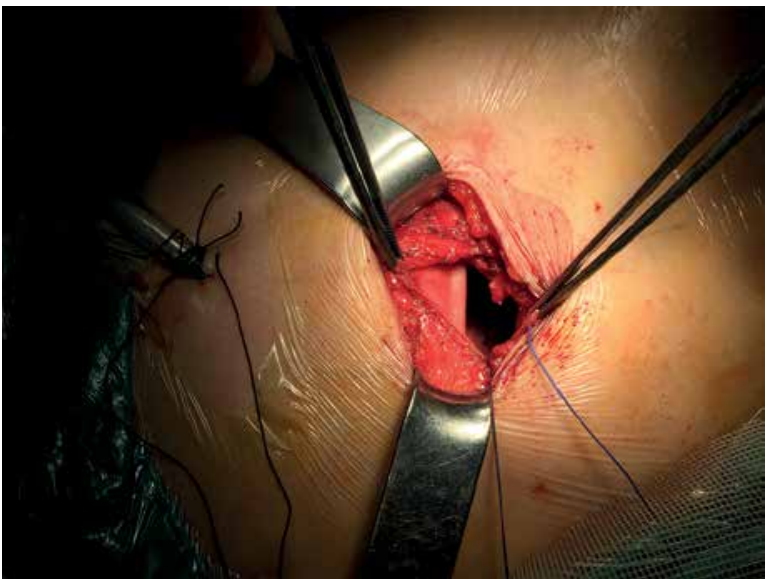

Photo 2. The lung was freed from adhesions and pulled into the pleural cavity

\section{Material and methods}

This study retrospectively analyses 650 (550 anatomic, 100 non-anatomic sublobar resections) patients who underwent lung resections for lung cancer in our department between 2012 and 2018. We detected lung herniation in 3 patients after VATS resection.

Demographic data and clinicopathological features were extracted from the medical records.

\section{Results}

\section{Case 1}

A 63-year-old man was admitted to our department with a complaint of chest pain and bulge in his chest wall. His medical history revealed that 1 year before, he underwent VATS right upper lobectomy because of non-small cell lung cancer with an incision at the level of the fourth intercostal space in the mid-axillary line. Chest computed tomography (CT) revealed protrusion of a small part of the right lower lobe through the fourth intercostal space (Photo 1). Surgical repair of lung herniation was planned. After a surgical incision on the fourth right intercostal space in mid-axillary line, the herniated lung parenchyma appeared adhering to the parietal pleura and to the external chest strap. The lung was freed from adhesions and pulled into the pleural cavity (Photo 2). The fourth intercostal space was closed tightly with 2/0 Vicryl sutures and the thoracic wall was reinforced using a polytetrafluoroethylene patch (Photo 3). The post-operative course was uneventful and follow-up CT at 7 months after surgery showed no recurrence of hernia.

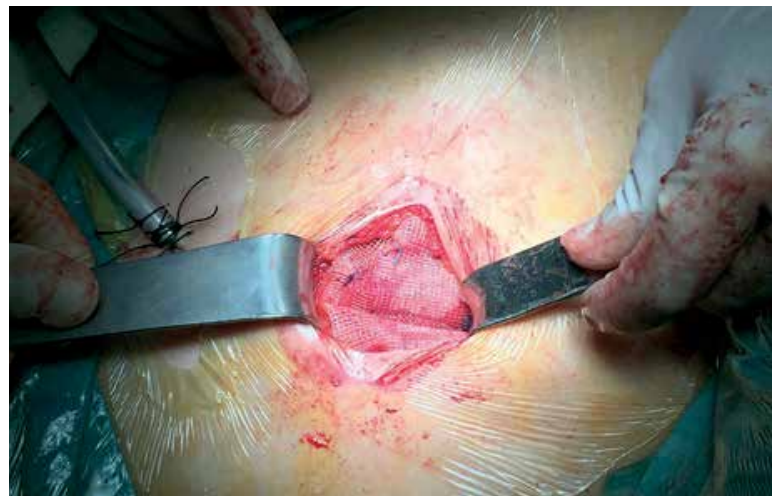

Photo 3. The fourth intercostal space was closed tightly with $1 / 0$ Vicryl sutures and the thoracic wall was reinforced using a polytetrafluoroethylene patch 


\section{Case 2}

A 65-year-old man was referred to our department for chest pain and a bulge on his chest. 3 months before he underwent re-operation for metachronous lung cancer and VATS wedge resection was performed. An incision at the level of the fourth intercostal space in the mid-axillary line was seen in physical examination. Chest CT revealed enlargement of the $4^{\text {th }}$ intercostal space and protrusion of the lung parenchyma. Surgical repair of lung herniation was planned. The fourth intercostal space was closed tightly with $2 / 0$ Vicryl suture but this time because of the enlarged intercostal space we preferred to use a Gore-Tex patch to reinforce the thoracic wall. The post-operative course was uneventful and follow-up CT at 7 months after surgery showed no recurrence of hernia.

\section{Case 3}

A 58-year-old woman was admitted to our department with chronic cough and chest pain. Her medical history revealed uniportal VATS wedge resection for a nodule in the left lung before 10 months. A $3 \mathrm{~cm}$ port incision was seen at the level of the $7^{\text {th }}$ intercostal space in the posterior axillary line. Chest CT revealed protrusion of a small part of the left lower lobe through the $7^{\text {th }}$ intercostal space (Photo 4). Surgical repair of the lung herniation was planned. Ribs were approximated after the lung was pulled into the pleural cavity. The thoracic wall was reinforced using a polytetrafluoroethylene patch. The post-operative course was uneventful.

In these cases, polytetrafluoroethylene and Gore-Tex patches were used depending on the surgeon's personal preference. There was no recurrence in the follow-up period and all patients of this series recovered uneventfully (Table I).

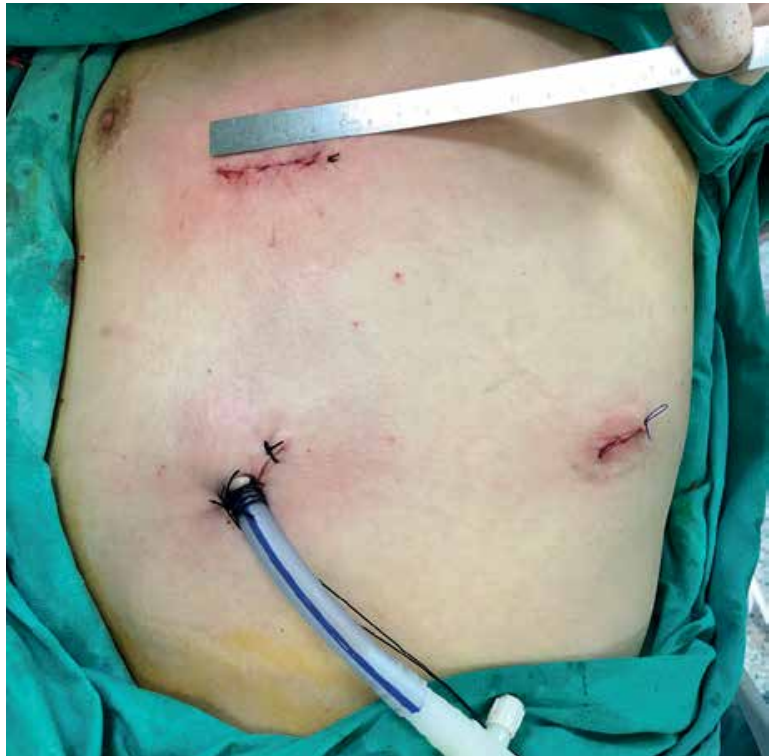

Photo 4. This image belongs to a patient who has undergone VATS lobectomy. We usually make the utility" incision $(3$ to $6 \mathrm{~cm}$ ) in the anterior axillary line, about $5^{\text {th }}$ intercostal space

\section{Discussion}

Lung herniation is a rare condition and it can be classified as congenital and acquired. The acquired cases are split into traumatic, spontaneous, and pathological. According to the literature, most of the cases are traumatic [9]. Increased intrathoracic pressure and weakness of intercostal muscles are the main pathology of lung herniation. Postoperative lung hernias are mostly reported as a complication of anterior thoracotomy in the literature [10]. The thoracic cage has an "inherent weakness" anteriorly, and the anterior and inferior aspects of the intercostal space were relatively wide. So these anatomic conditions increase the risk of lung herniation in anterior thoracotomies. In the video-assisted thoracoscopic surgery procedure a "utility"

Table I. Patient characteristics, symptoms and used materials are seen in the table

\begin{tabular}{|lccccccc|}
\hline Case & Gender & Age & Operation & Symptom & Used material & $\begin{array}{c}\text { Follow-up } \\
\text { [months] }\end{array}$ & \begin{tabular}{c} 
Recurrence \\
\hline 1
\end{tabular} \\
\hline 2 & 63 & $\begin{array}{c}\text { Lobectomy } \\
\text { (RUL-Triportal) }\end{array}$ & Chest pain and bulge & Polytetrafluoroethylene & No \\
\hline 3 & M & 65 & $\begin{array}{c}\text { Wedge } \\
\text { (Uniportal) }\end{array}$ & Chest pain and bulge & Gore-Tex & 7 & No \\
\hline
\end{tabular}




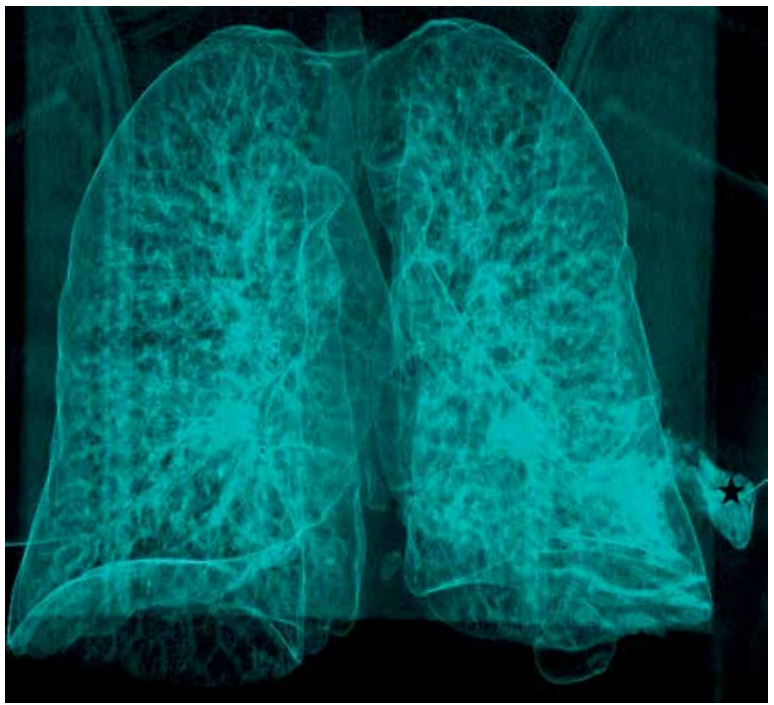

Photo 5. Three-dimensional volume rendering of CT data can provide additional benefit to the visualization of the herniated parenchyma (asterix)

incision (3 to $6 \mathrm{~cm}$ ) is usually placed in the anterior axillary line, about the 5th intercostal space [11]. For convenience purposes, many surgeons prefer to make a longer incision under the skin especially in the intercostal space. These factors, which are specific to the VATS procedure, contribute additional risk to lung herniation. For lung herniation some predisposing factors are defined in the literature: activities that increase intrathoracic pressure such as cough and heavy lifting, chronic obstructive pulmonary disease, inflammatory or neoplastic processes and chronic steroid usage [12].

According to the literature in most cases patients usually present with chronic chest pain and a bulge on the chest wall. Chest radiography and thorax computed tomography both provide valuable information. Three-dimensional volume rendering of CT data can provide additional benefits (Photo 5).

Asymptomatic lung hernias may be managed by close observation but because of the risk of incarceration of the pulmonary parenchyma, surgery is often necessary.

The main steps of treatment involve: identification of the hernia, freeing of all adhesions, reduction of the lung tissue back into the thoracic cavity and repairing the defect of the chest wall.

Different kinds of surgical techniques to repair the defect have been reported previously. We prefer to use Vicryl for approximation of ribs in addition to an intrathoracic polytetrafluoroethylene patch (in 2 cases) and Gore-Tex mesh (in 1 case). Autologous tissue usage for repair was also reported in the literature [7].

According to our experiences and the relevant literature we defined some risk factors for lung herniation after the VATS procedure:

1. Opening an intercostal space larger than the skin incision. Although opening a large intercostal space is an inevitable condition, especially in the presence of large tumors, the incision should be kept as small as possible.

2. Significant emphysema and chronic cough especially in patients with chronic obstructive pulmonary disease (COPD).

3. Weakness of the thoracic muscles.

4. Non-rigorous closure of the tissue layers.

Prevention is the best method in order to avoid a lung hernia. So, we recommend:

1. Making a small intercostal incision if possible.

The use of endoscopic instruments instead of conventional ones could eliminate the need for wide incisions.

2. Approximating the intercostal space.

Especially when the intercostal opening is significantly larger than the skin incision. We routinely apply rib approximation in patients with significant emphysema and large tumor.

3. Suturing each layer separately (the intercostal and pectoralis muscles and additionally deep dermal and subcuticular layers).

While intercostal muscle is being incised, the distance to allow suturing should be maintained. 4. Avoiding unnecessary soft tissue dissection.

\section{Conclusions}

Lung herniation is a rare complication of VATS and it was mostly reported as a complication of anterior thoracotomy in the literature. Nowadays the VATS procedure has become the dominant method of lung cancer surgery but there is no case series about the complications of lung herniation in the literature. By this retrospective case series, we defined some patient-related and surgeon-related risk factors and some basic recommendations for prevention of this complication.

\section{Conflict of interest}

The authors declare no conflict of interest. 


\section{References}

1. Patterson GA, Cooper JD, Deslauriers J, et al. Pearson's Thoracic \& Esophageal Surgery. $3^{\text {rd }}$ edition. Churchill Livingstone Elsevier, Philadelphia 2008; 8: 1770.

2. Weissberg D, Refaely Y. Hernia of the lung. Ann Thorac Surg 2002; 74: 1963-66.

3. Bousson V, Arrive L, Brauner M. Lung herniation occurring after video-assisted thoracic surgery. AJR Am J Roentgenol 1999; 172: 1145-6.

4. Hauser M, Weder W, Largiader F, Glazer GM. Lung herniation through a postthoracoscopy chest wall defect: demonstration with spiral CT. Chest 1997; 112: 558-60.

5. Ishibashi H, Hirose M, Ohta S. Lung hernia after video-assisted thoracoscopic lobectomy clearly visualized by three-dimensional computed tomography. Eur I Cardiothorac Surg 2007; 31: 938.

6. Temes RT, Talbot WA, Green DP, Wernly JA. Herniation of the lung after video-assisted thoracic surgery. Ann Thorac Surg 2001; 72: 606-7.

7. Johnson C, Weksler B. Lung hernia after video-assisted thoracoscopic lobectomy. Innovations 2012; 5: 300-2.

8. Ema T, Funai K, Kawase A, et al. Incarceration hernia of the lung after video-assisted thoracic surgery requiring emergency operation: a case report. J Thorac Dis 2018; 10: E541-3.

9. Forty J, Wells FC. Traumatic intercostal pulmonary hernia. Ann Thoracic Surg 1990; 49: 670-1.

10. Athanassiadi K, Bagaev E, Simon A, Haverich A. Lung herniation: a rare complication in minimally invasive cardiothoracic surgery. J Thorac Cardiovasc Surg 2008; 33: 774-6.

11. He J, Xu X. Thoracoscopic anatomic pulmonary resection. J Thorac Dis 2012; 4: 520-47.

12. Munnell ER. Herniation of the lung. Ann Thorac Surg 1968; 5: 204-12.

Received: 16.04 .2019 , accepted: 4.06.2019 